Ann. Biol. anim. Bioch. Biophys., 1969, 9 (I), I23-134.

\title{
MÉTHODES D'ANALYSE DE LA STRUCTURE MUSCULAIRE
}

\author{
O. SCHMITT, B.-L. DUMONT \\ avec la collaboration technique de Claudette Chevalier et Thérèse DrRine
}

Laboratoire de Recherches sur la Viande,

Centre national de Recherches zootechniques, 78-Jouy-en-Josas

Institut national de la Recherche agronomique

\section{SOMMAIRE}

Il est présenté une méthode d'étude de la structure du muscle, définie par l'arrangement des divers types de faisceaux de fibres qui le constituent. Cette méthode fait appel à une technique d'obtention de sections de muscle de grande surface (jusqu'à plusieurs centaines de $\mathrm{cm}^{2}$ ). Le fixateur utilisé sur des échantillons préalablement congelés est un mélange à volume égal d'alcool absolu, d'acide acétique et de chloroforme employé dans un milieu turbulent obtenu par agitation magnétique. Il est préconisé, après fixation et lavage par l'alcool, d'imprégner le tissu musculaire par de la glycérine et d'effectuer l'inclusion sous vide partiel.

Les difficultés susceptibles d'être rencontrées dans l'exécution pratique sont exposées et des remèdes proposés.

Dans l'ensemble, la méthode assure une bonne fixation du tissu musculaire et du conjonctif, et entraîne une rétraction de la surface des échantillons de l'ordre de $30 \mathrm{p}$. I00 en moyenne.

Grâce à cette technique il est possible de considérer de grandes surfaces de coupe, de faible épaisseur (IO-I $5 \mu$ ), observées sur écran ou après agrandissement photographique:-

D'autre part, il est suggéré d'envisager de déterminer la structure du muscle en considérant les caractéristiques des unités d'organisation - appelées Myoskhènes - que paraît révéler l'examen de la trame conjonctive relativement homogène qui sépare certains groupes de faisceaux lorsqu'on examine la coupe d'un muscle à un très faible grossissement $(<4)$. Les dimensions moyennes des myoskhènes, l'importance de leur périmysium, le nombre et la nature des groupes de faisceaux de fibres qu'ils renferment sont proposés comme autant de critères propres à définir de façon quantitative la structure des muscles. Il est décrit un mode d'estimation subjective de la dimension et de l'importance du périmysium des myoskhènes et un mode objectif de jugement de la nature des groupes de faisceaux, exprimant un degré de structuration du muscle.

\section{INTRODUCTION}

Ire cloisonnement du muscle en faisceaux de fibres d'inégale importance est une notion classique de la myologie, connue de longue date (BouRGELAT, I797). Son étude a, jusqu'ici, essentiellement précisé (WALLS, I960) les caractéristiques des fibres musculaires - dimension, forme - par la mise en œuvre de techniques simples, portant sur des échantillons de taille réduite. La structure du muscle - telle qu'on peut la définir à partir de la dimension et de l'arrangement des divers fais- 
ceaux qui en déterminent ce qu'il est convenu d'appeler la texture, n'est connue que très imparfaitement. Son analyse n'a pas donné lieu à des recherches approfondies, sauf en ce qui concerne, pour de très petits muscles, le nombre total de fibres. En particulier les rapports existant entre la nature de la trame du tissu conjonctif et le développement des fibres ne paraissent pas élucidés.

La difficulté d'obtention de coupes histologiques de surface suffisante, offrant à considérer un vaste champ, a certainement été le facteur limitant des possibilités d'analyse de la structure musculaire. Le présent travail décrit une teshnique d'étude de dernière reposant, d'une part, sur l'obtention de sections de muscle de grande surface et de faible épaisseur et, d'autre part, sur l'appréciation quantitative de la structure du muscle.

\title{
I. - TECHNIQUE HISTOLOGIQUE D'OBTENTION \\ DE SECTIONS DE MUSCLE DE GRANDE SURFACE
}

\author{
EXPOSÉ DE LA TECHNIQUE
}

L'obtention de coupes sériées de faible épaisseur - telles que les néces'itent les travaux intéressant la structure musculaire conduits dans notre laboratoire -- impose le recours à l'inclusion à la paraffine. Ce mode d'inclusion offre, d'autre part, l'avantage de permettre la conservation pratiquement illimitée des échantillons.

Dans des essais anterieurs, nous avions mis en évidence que, parmi les différentes méthodes classiques de fixation, la technique utilisant le mélange de Gilson comme fixateur se révélait intéressante pour la préservation des fibres musculaires et du tissu conjonctif (DumONT et PARIs, I959 a), dans le cas d'échantillons de faible taille (20 à $25 \mathrm{~mm}$ de long sur 3 à Io $\mathrm{mm}$ d'épaisseur). Au-delà de ces dimensions, l'application de cette technique conduisait à des difficultés conjointes de coupe et de mauvaise fixation, qui imposaient comme limite d'épaisseur une épaisseur maximum de ro mm. Bien que déjà importante - à l'echelle microscopique - la surface musculaire observable dans ces conditions nous apparaissait, à l'usage, très insuffisante en raison de l'hétérogénéité constatée d'un échantillon à l'autre d'un même muscle et des difficultés, sinon des impossibilités, de reconstitution de l'ensemble de la surface d'un muscle ou même simplement de la surface correspondant aux quantités de muscle consommées lors de la mastication d'une bouchée de viande.

La nécessité d'accroître la surface des échantillons impliquait une augmentation très sensible de leur épaisseur pour assurer une adhérence latérale suffisante au bloc de paraffine lors de la coupe. Cette augmentation de l'épaisseur posait des problèmes en ce qui concerne la fixation, compte tenu des vitesses de pénétration du fixateur en milieu normal (de l'ordre de $\mathrm{I} \mathrm{mm} / \mathrm{heure}$ ). Pour obtenir une homogénéité de fixation il était indispensable de pouvoir accélérer la pénétration du fixateur. A cette fin, nous avons eu recours à une fixation de l'échantillon immobilisé dans un milieu turbulent, obtenu par agitation magnétique.

Lors des premiers essais le mélange fixateur utilisé fut identique à celui précédemment employé (Dumont et PARIS, I959 a) qui supposait, après fixation, l'élimination du sublimé par l'iode. Cette dernière opération entraînait, dans le cas de gros échantillons, des difficultés, provoquant notamment des ruptures des pièces. Cette observation nous conduisit à supprimer le bichlorure de mercure de la formule du fixateur et de la simplifier en ne conservant que le mélange à volume égal d'alcool absolu, d'acide acétique cristallisable et de chloroforme. Cette suppression n'entraînait pas une modification notable de la qualité du fixateur mais éliminait tous les inconvénients provoqués par la présence du sublimé.

La dureté naturelle des échantillons (spécialement ceux riches en conjonctif) jointe à la grandeur des pièces à couper nous a conduits à modifier le cycle habituel des préparations, entre la fixation et l'inclusion dans la paraffine. Après l'élimination du fixateur, nous avons procédé à une imprégnation. des tissus par la glycérine, préconisée par LEPLAT (I939) pour les objets durs à couper.

La nécessité de pouvoir considérer un grand nombre de muscles du même animal nous a amenés à envisager une congélation rapide préalable des pièces. Le but initial de cette pratique était la conservation. En plus nous devons noter que la congélation permet de rectifier facilement la surfare 
ct l'épaisseur des échantillons et d'en obtenir, de façon simple, un contour précis. Toutefois, dans le cas d'un échantillon isolé pour lequel ne se pose pas de problèmes de conservation, la congélation n'est pas nécessaire. Le tableau I indique les différentes opérations de la mise en œuvre de la méthode, ainsi que des observations complémentaires intéressant des cas particuliers ou des détails pratiques de la technique.

\section{INSUCCÈS ETT REMÈDES}

Au cours des travaux qui ont conduit à l'élaboration de cette technique, nous avons enregistré un certain nombre de difficultés qui sont susceptibles de se produire aux différentes manipulations. Nous présentons dans le tableau 2 les remèdes proposés pour surmonter les échecs ou les insuccès éventuels.

\section{RÉSULTATS ET DISCUSSION}

La méthode permet sous une faible épaisseur ( $5 \mu$ environ) l'obtention de coupes de très grandes surfaces $(20 \times$ ro $\mathrm{cm})$, ce qui peut intéresser des muscles entiers de nombreux types de mammifères ou des portions importantes des muscles les plus volumineux d'animaux de très grande taille. Ainsi dans le cas du bouf la quasi-totalité de la musculature est justiciable de cette technique sans qu'il soit besoin de fragmenter les muscles pour l'inclusion. De ce fait la méthode ouvre de larges horizons d'étude de la structure musculaire.

Dans l'ensemble la fixation des fibres musculaires et des éléments de la trame du tissu conjonctif est satisfaisante et homogène ; ce qui est en accord avec les observations précédentes concernant l'intérêt du mélange de Gilson par rapport à d'autres types de fixateurs tels le formol, le Bouin, le Carnoy, comme fixateur du tissu musculaire, dans le cas de petits échantillons (Dumont et PARIS, I959 b). Il en va de même pour ce qui concerne la facilité de coupe qui est même nettement améliorée par rapport à ce qu'elle est avec des échantillons de petite taille.

L'aisance de la coupe, la possibilité de programmation des différentes opérations font de cette méthode une technique susceptible d'être utilisée en travail de routine pour des études d'examen systématique de coupes sériées examinées sous faible épaisseur, ce qui lui confère une supériorité certaine sur les autres techniques disponibles jusqu'ici (congélation, celloïdine, ...).

La présence à l'intérieur de certains muscles - eg. supraspinatus ou infraspinatus - de grandes lames aponévrotiques peut toutefois gêner en partie la réussite de cette technique, en perturbant la coupe lorsque le rasoir attaque l'aponévrose interne dans sa plus grande dimension. On peut y remédier soit en orientant latéralement le rasoir, au moment de la coupe, soit en orientant l'échantillon lors de l'inclusion.

La manipulation d'échantillons de grandes dimensions pose des problèmes, sur le plan matériel, l'équipement classique des laboratoires d'histologie étant en règle générale conçu pour des pièces de taille réduite. Nous insisterons surtout ici sur la nécessité d'utiliser un microtome dont la hauteur de coupe soit suffisante et qui soit très stable. En pratique seuls les grands microtomes sur pied, très lourds, peuvent convenir.

L'observation au microscope optique est longue et même impossible au-delà de la taille maxima permise par les plus importantes platines. Il est nécessaire pour 


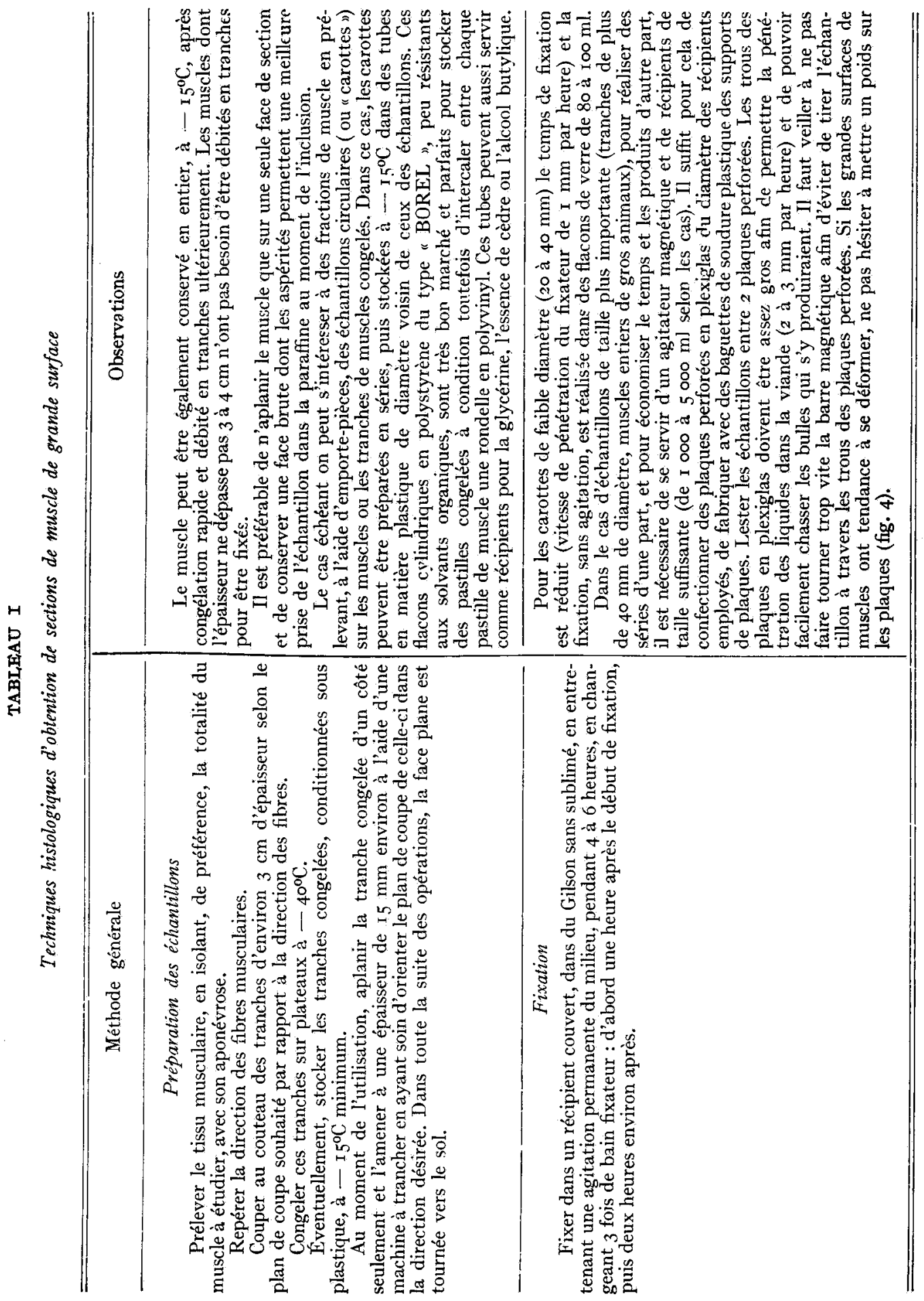




\begin{tabular}{|c|c|c|c|c|c|c|c|c|}
\hline & 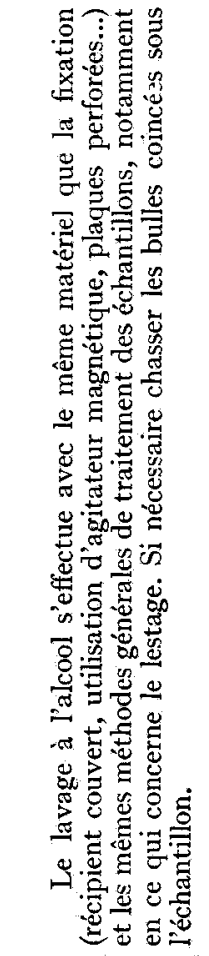 & 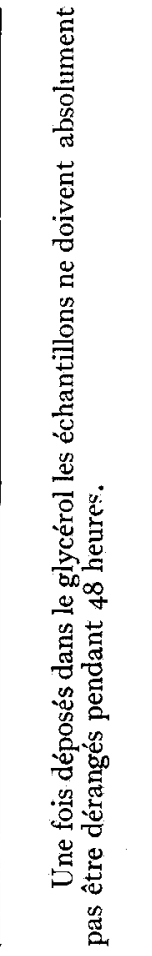 & 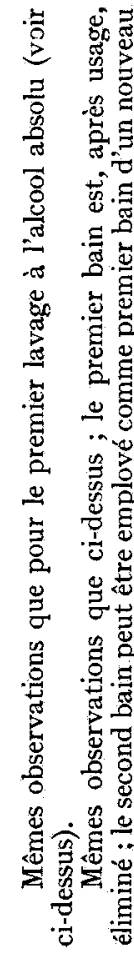 & 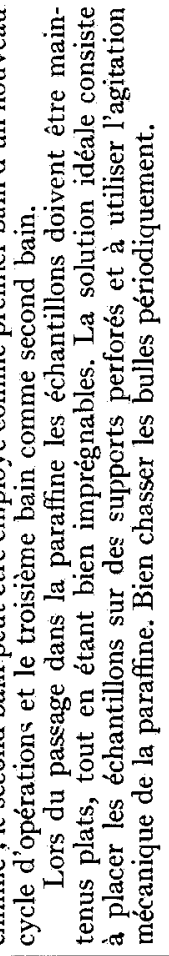 & 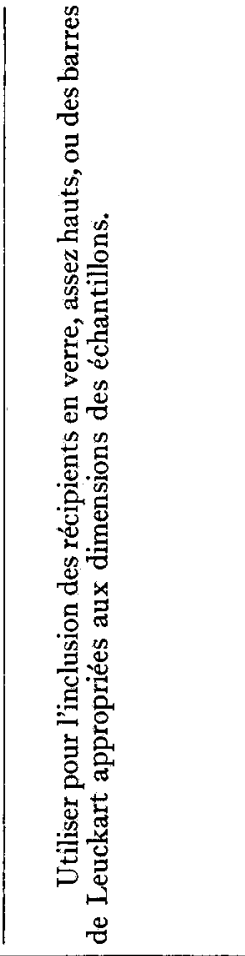 & & 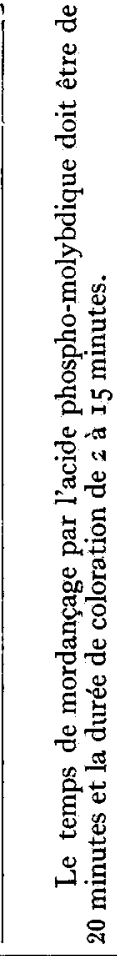 & 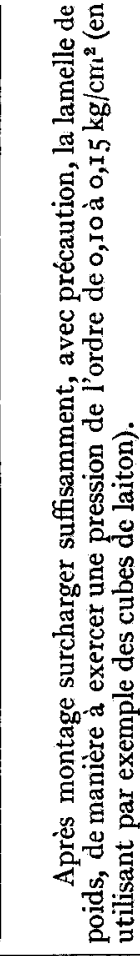 \\
\hline 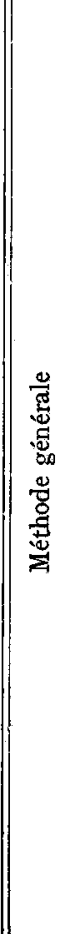 & 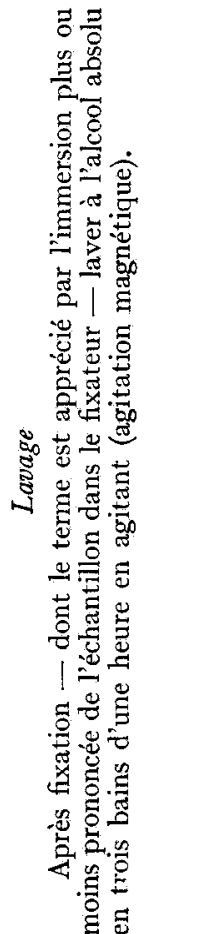 & 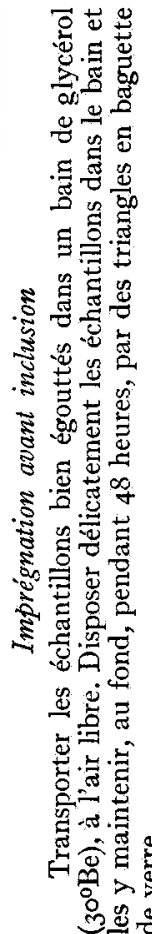 & 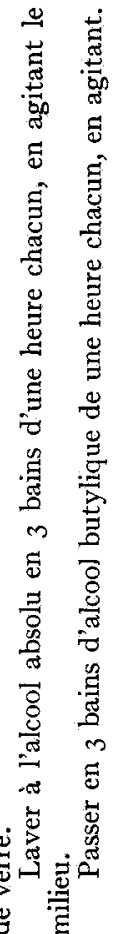 & 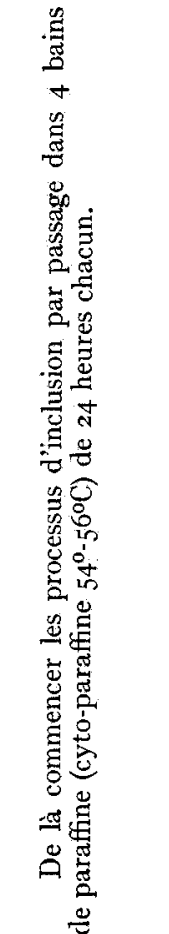 & 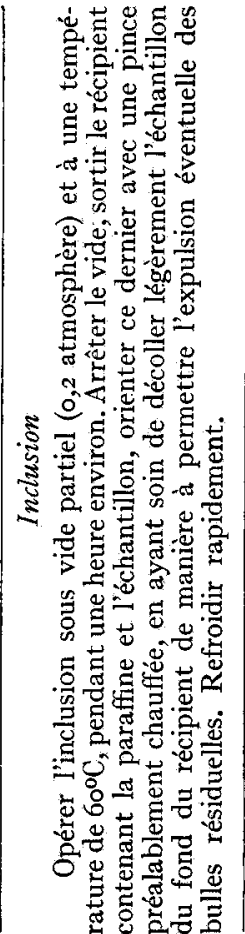 & 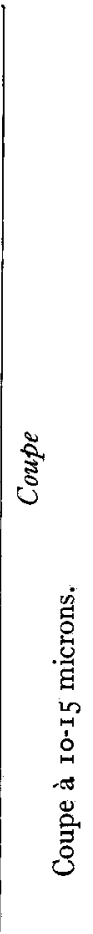 & 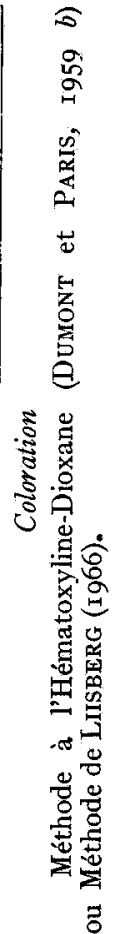 & 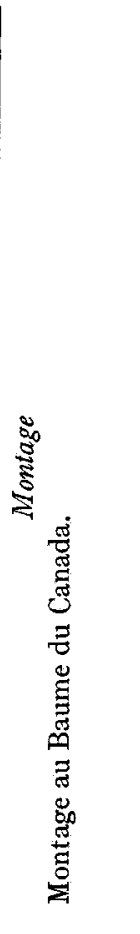 \\
\hline
\end{tabular}




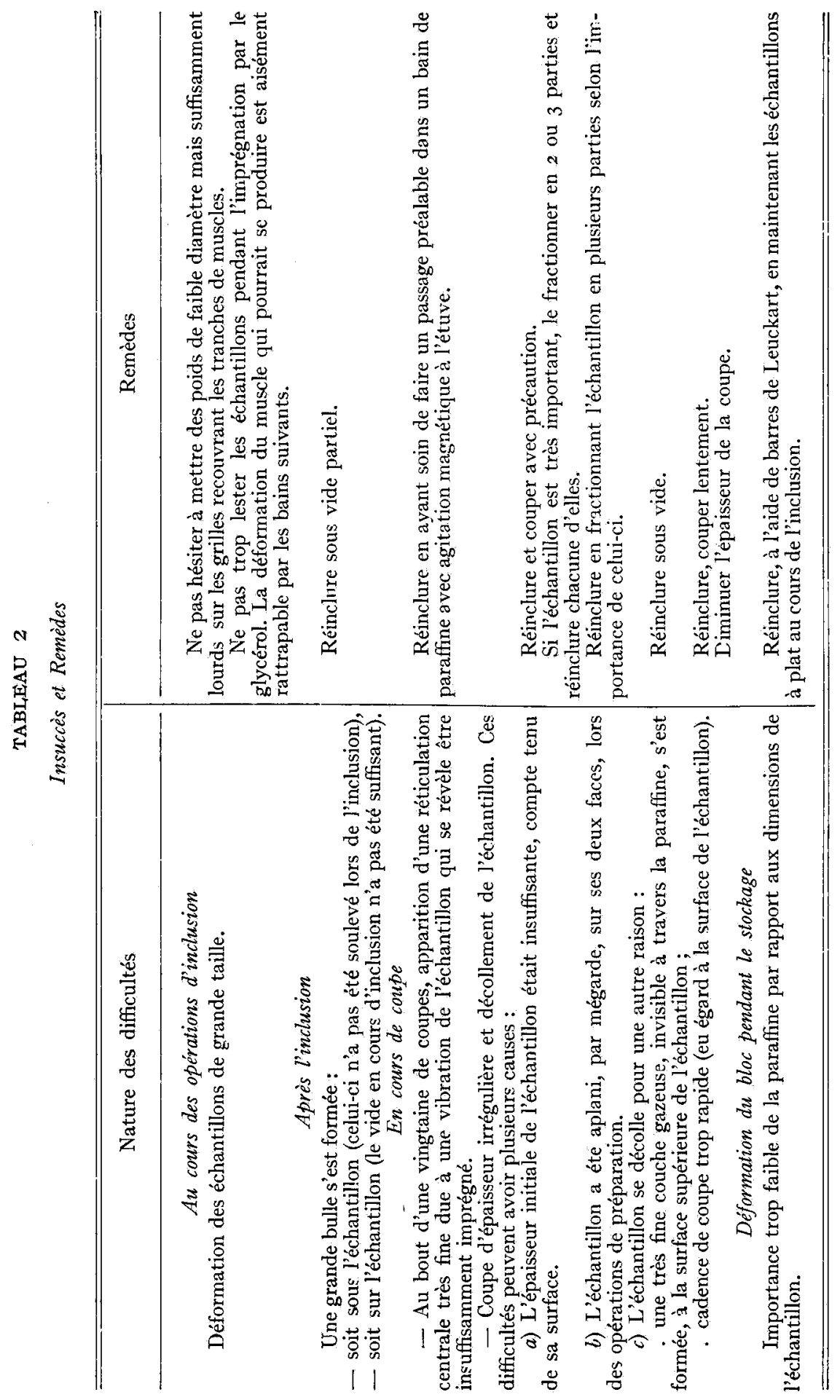


éviter la fatigue des yeux d'observer les grandes coupes soit par projection directe de la préparation sur écran ou sur verre dépoli, soit, mieux, d'utiliser des techniques photographiques. Dans ce cas on peut employer la préparation directement dans un agrandisseur et obtenir un négatif sur papier. On peut également utiliser du plan film - de type Graphic Tone - pour obtenir des épreuves par contact ou des négatifs destinés à être projetés pour fournir des positifs géants, à l'agrandissement souhaité.

D'autre part la consommation des différents produits est assez importante en valeur absolue (quoique relativement plus faible en considérant les volumes des échantillons que dans le cas de traitement de pièces de petite taille).

A l'issue de la préparation l'échantillon a subi par rapport à l'état frais une rétraction assez importante de son volume, spécialement par suite d'une diminution sensible de la surface. La rétraction est très variable d'un muscle à l'autre du même animal et entre animaux pour le même muscle. Cette rétraction finale marque le terme des variations de dimensions (hauteur et surtout surface) présentées par l'échantillon au cours des diverses phases de la préparation. A une légère augmentation se produisant au cours de la fixation fait suite une légère rétraction pendant le premier passage dans l'alcool absolu (ce qui ramène le volume de la pièce sensiblement au volume initial). Puis l'imprégnation par le glycérol se traduit par une forte diminution de la surface - dont la forme n'est pas cependant affectée -, qui est encore accentuée lors du passage dans les bains de paraffine.

Par rapport à la surface initiale on peut estimer qu'en moyenne la réduction constatée est de l'ordre de $30 \mathrm{p}$. Ioo, la marge de variation se situant, selon les muscles, entre I5 et $50 \mathrm{p}$. Ioo.

\section{II. - MÉTHODE D'APPRÉCIATION QUANTITATIVE}

DE LA STRUCTURE DU MUSCLE

En fournissant de grandes surfaces de tissu musculaire - et dans la plupart des cas en permettant de considérer la totalité de la section transversale d'un muscle, quelle que soit l'espèce zoologique - la technique que nous venons d'exposer permet d'aborder l'étude de la structure du muscle en considérant les rapports d'un très grand nombre de faisceaux de fibres musculaires de types différents.

L'observation d'un grand nombre de muscles d'origines différentes traités de cette façon nous conduit à suggérer la méthode suivante d'appréciation de la structure du muscle.

L'examen de la section transversale d'un muscle (perpendiculairement à la direction générale des fibres) laisse, en première analyse, l'impression d'une grande complexité d'agencement, quel que soit le grossissement considéré (fig. I). On remarque toutefois que cette complexité paraît s'accentuer au fur et à mesure qu'on examine la section à un plus fort grossissement et que se précise l'hétérogénéité du tissu musculaire traduite par une grande variabilité dans la forme et les dimensions des fibres, ainsi que par celles des faisceaux.

A faible grossissement ( $\times 2$ à 4 ), pour les petits muscles ou les muscles de taille moyenne, et même en grandeur nature pour les muscles assez importants, on constate, en considérant attentivement la coupe, l'existence, à côté de grandes travées conjonc- 
tives nettement orientées, d'une trame diffuse de tissu conjonctif qui paraît à peu près uniforme dans son épaisseur et relativement homogène dans sa répartition. Les contours de cette trame délimitent des surfaces d'importance et de forme parfois très variables mais qui ont, cependant, une allure générale propre à chaque muscle et qui semblent traduire une sorte de motif élémentaire de l'organisation et de la structure du muscle. Nous proposons d'appeler "MYOSKHÈNE " cette unité de structure musculaire et d'apprécier la structure générale du muscle dans sa section transversale d'après les caractéristiques moyennes des myoskhènes : dimensions, importance de leur périmysium, nombre et nature des groupes de faisceaux musculaires qu'ils renferment.

Les fibres musculaires sont groupées en faisceaux dits faisceaux primaires, que nous appellerons ici faisceaux d'ordre $I$. Chacun de ces faisceaux est entouré par un périmysium de faible importance. Un nombre variable de faisceaux d'ordre I peuvent être regroupés pour constituer un faisceau de beaucoup plus grande taille ou faisceau d'ordre 2, délimité par un périmysium nettement plus marqué que celui des faisceaux d'ordre I. On peut considérer qu'à leur tour un certain nombre de faisceaux d'ordre 2 sont assemblés pour donner un faisceau d'ordre 3, au périmysium encore plus important, etc. Finalement un ensemble de faisceaux d'ordre $n$ - I constitue un myoskhène d'ordre $n$. La valeur de l'ordre du myoskhène traduit ce que nous appellerons le degré de structuration du muscle.

On` peut suggérer d'utiliser une échelle d'évaluation subjective pour la moyenne des dimensions des myoskhènes, ainsi que pour évaluer l'importance de leur périmysium. A défaut d'une échelle universelle susceptible d'être employée pour tous les muscles, quelle que soit l'espèce ou l'âge des individus, - échelle qui reste à établir nous utilisons, temporairement, une échelle particulière d'évaluation, propre à chaque expérience, employée à titre comparatif et tenant compte de la variabilité constatée entre échantillons extrêmes.

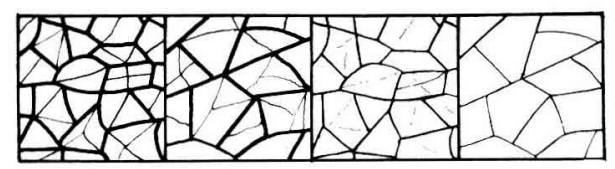

a) - Valeurs décroissantes de la note d'estimation de l'importance du tissu conjonctif; de gauche à droite respectivement $8,6,4$ et 2 .

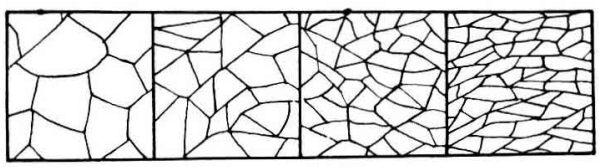

b) - Taille des myoskhènes correspondant respectivement de gauche à droite à des notes de 8,6 , 4 et 2 dans l'échelle d'évaluation.

FIG. 2. - Illustration schématique, a faible grossissement, des échelles d'évaluation de la taille des myoskhenes et de l'importance de leur périmysium 


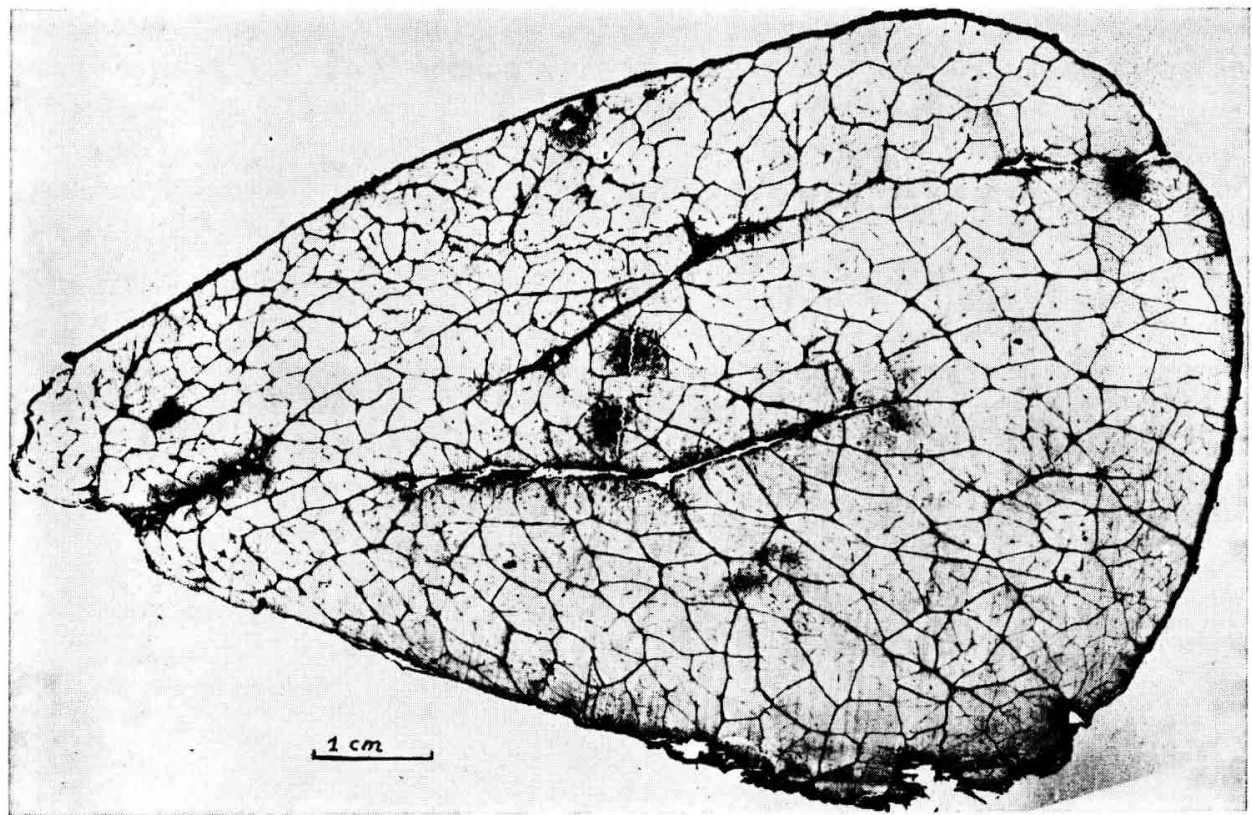

Fig. I. - Section transversale du muscle semi-tendinosus de Bovin (Bos Taurus) Veau Charollais de 3 mois

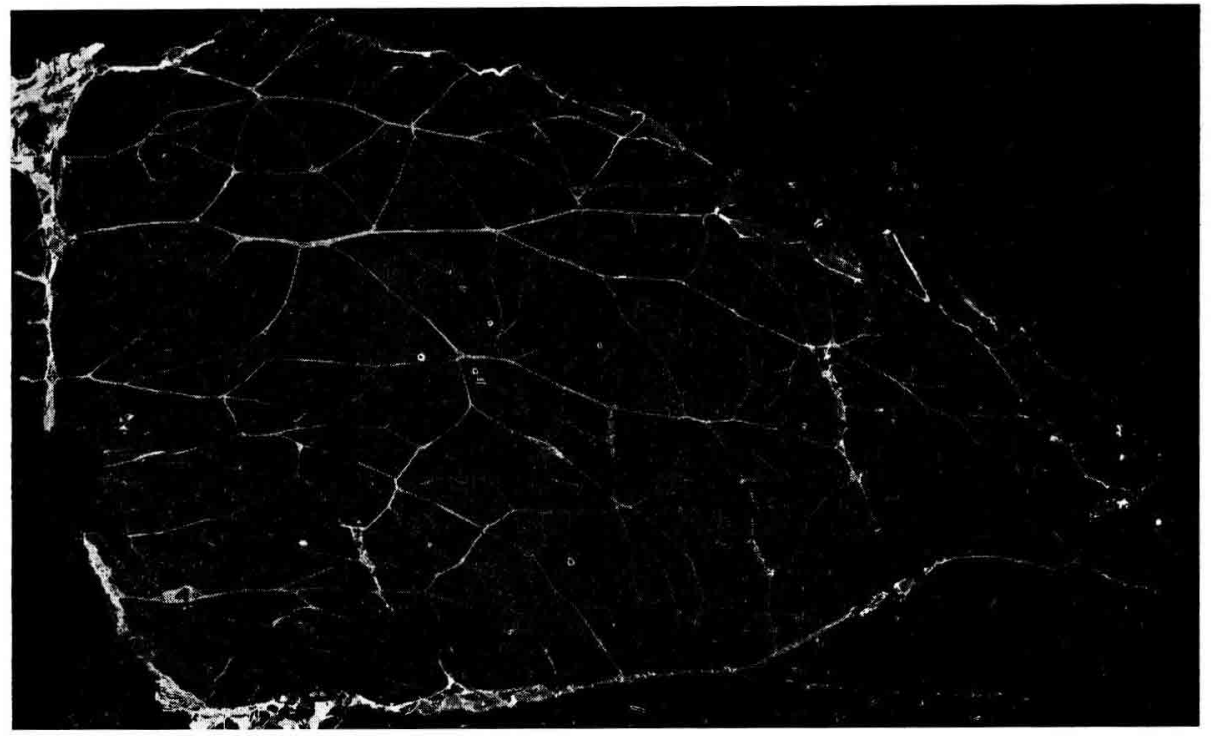

Fig. 3. - Section transversale de muscle Rhomboìdeus de Bovin (Bos Taurus) Taurillon Limousin $\times$ Charollais de 9 mois 
La figure 2 donne, à titre d'exemple, une illustration schématique des échelles d'évaluation de la taille des myoskhènes et de l'importance de leur périmysium retenues dans une étude comparative de muscles de porcins de Ioo $\mathrm{kg}$ de poids vif. La figure 3 (Rhomboïdeus) montre les limites de la signification qu'il faut accorder à la notion de " moyenne de dimension " et $d$ ' importance du périmysium " dans un muscle donné. Malgré le caractère subjectif de l'appréciation de ces facteurs, l'expérience pratique nous a montré l'utilité de cette méthode dans des tests de comparaison (BOCCARD et al., ig67).

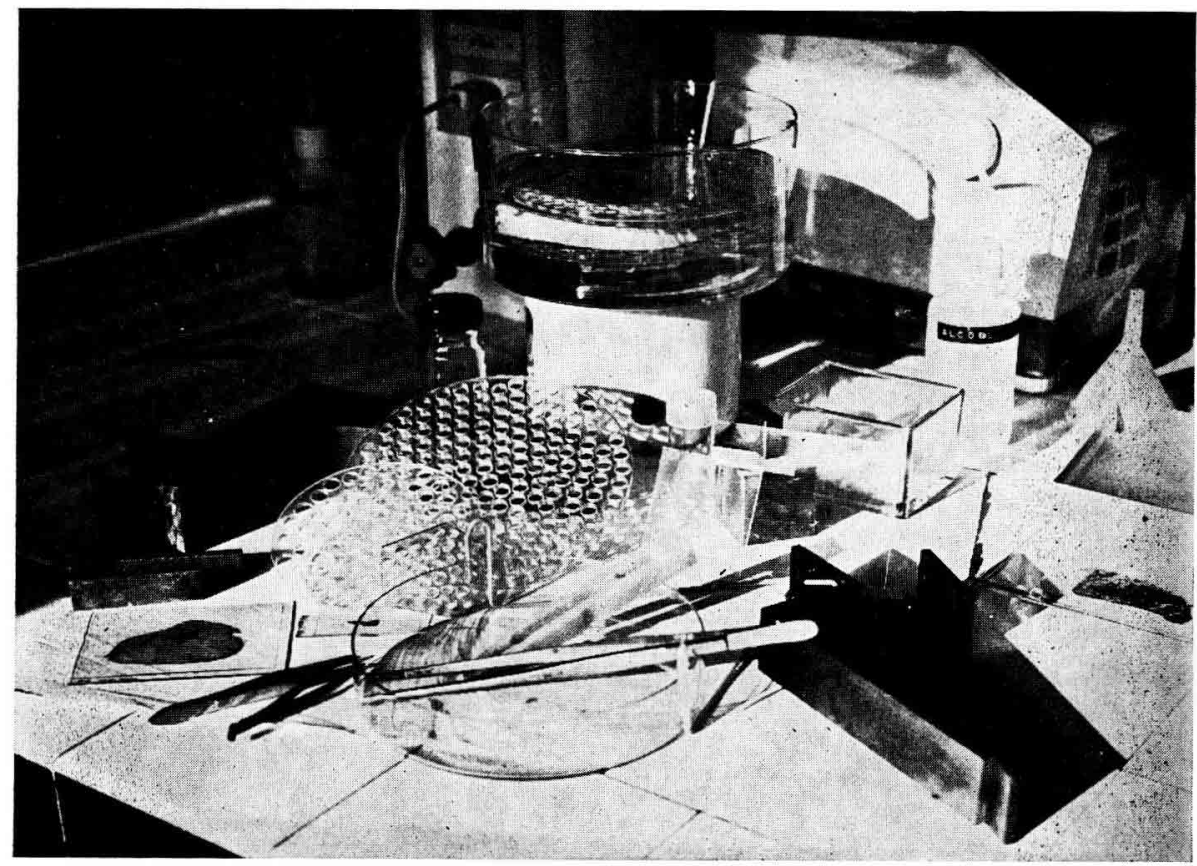

Fig. 4. - Partie du matériel histologique nécessaire pour obtenir de grandes surfaces musculaires.

Le degré de structuration est une notion plus facilement quantifiable dans la mesure où on peut le définir à partir de la moyenne des numéros d'ordre de $n$ myoskhènes d'un muscle donné. Ainsi, par exemple, dans le cas de l'étude comparative des muscles de porcs déjà mentionnée, le degré de structuration du muscle Teres major s'établissait pour 32 myoskhènes à 4,37 avecla répartition suivante des numéros d'ordre:

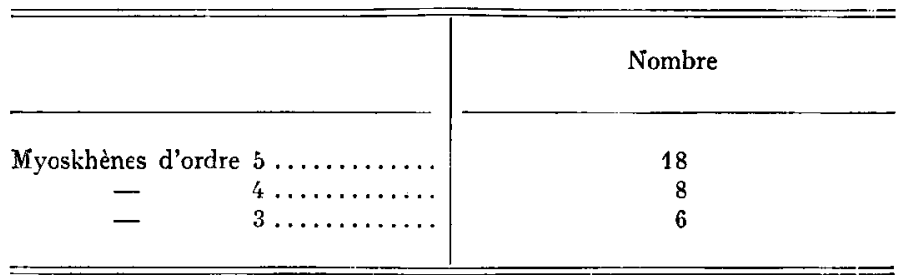

pour un total de 87 faisceaux primaires, d'ordre $I$. 
Un mode plus rapide d'estimation - mais moins précis - de la structuration peut consister à déterminer le nombre d'ordre existant dans un myoskhène donné par appréciation du nombre des divers types de périmysium qu'il renferme. Pour cela on considère, à l'intérieur du myoskhène, d'abord le périmysium délimitant un faisceau d'ordre $n-\mathrm{I}$, puis à l'intérieur de ce faisceau le périmysium délimitant un faisceau d'ordre $n-2$, etc. En fin de comptage, on aboutit à un dernier type de périmysium qui correspond à l'enveloppe conjonctive d'un faisceau primaire. Le nombre de différents types de périmysium rencontrés indique l'ordre du myoskhène. En répétant cette estimation sur une dizaine de myoskhènes différents et en retenant la moyenne, on obtient une approximation suffisante du degré de structuration du muscle considéré.

Finalement, les valeurs attribuées par la méthode que nous proposons aux 3 paramètres des myoskhènes - taille, importance du périmysium et degré de structuration - d'un muscle donné, permettent selon nous une description satisfaisante de la structure de ce muscle.

Il est bon de rappeler, en outre, que la technique d'obtention de sections de muscle de grande surface permet de déterminer également le nombre des fibres musculaires de chaque faisceau primaire.

Le traitement statistique qu'autorise la quantification de ces différents caractères devrait offrir des perspectives intéressantes pour la connaissance du déterminisme biologique et des conséquences technologiques du développement musculaire des animaux domestiques.

Reçu pour putlication en acĥt Igt8.

\section{SUMMARY}

\section{METHODS OF ANALYSIS FOR MUSCULAR STRUCTURE}

The current paper describes a method for studying the structure of muscular tissue, according to the arrangement of the various kinds of bundles. The technique (table $r$ ) allows to obtain thin cuts (Io to I $5 \mu$ ) from a large area (up to $400 \mathrm{~cm}^{2}$ ). After freezing, the muscle is fixed in a magneticfanned mixture of absolute alcohol, acetic acid and chloroform. After alcohol washing, the muscle is impregnated in glycerin and included under partial vacuum.

The practical difficulties of the technique and their possible solutions are described in table 2.

On the other hand, it is suggested to describe the texture of muscles according to the characteristics of muscular structure units termed "Myoskhènes". These units can be defined starting from the reticulum of connective tissue sheathing some bundles, as it can be seen at very low magnification $(<\times 4)$ (fig. I). The average size of " myoskhenes ", the importance of their perimysium, the type and number of fiber bundles they include, appear to be the most relevant criteria to quantitatively define muscular structure. Methods for judging these characteristics are proposed either subjectively (ffg. 2) for the size of myoskhènes and perimysium, or objectively for the arrangement of different kinds of fiber bundles, which are in relation with the structuration degree of the muscle.

\section{RÉFÉRENCES BIBLIOGRAPHIQUES}

Boccard R., Dumont B.-L., Schmitt O., I 967 . Note sur les relations entre la dureté de la viande et les principales caractéristiques du tissu conjonctif. $13^{\mathrm{e}}$ Réunion des Instituts de Recherches sur les Viandes. Rotterdam, I $967(23$ p.).

Bourgelat C., 1797. Éléments de l'art vétérinaire. Librairie vétérinaire de la citoyenne Huzard, Paris. Dumont B.-L., Paris J., I959 a. Application des méthodes de coloration différentielle du tissu musculaire 
et du tissu conjonctif aux travaux de recherches sur la Viande. $\boldsymbol{5}^{\mathrm{e}}$ Réunion des Instituts de Recherches sur les Viandes. Paris ( $14 \mathrm{p}$.).

Dumont B. L., Pakis J., 1959 b. Étude critíque de différents fixateurs du tissu musculaire. $5^{\circ}$ Réunion des Instinuts de Recherches sur les Viandes. Paris (i 2 p.).

LePlat G., 1939. In Précis de Mitroscopie de M. Langeron, Masson Éditeurs, Paris, I949.

LIISBERG M. F, I966. A new and easy staining method for collagen and reticular fibres. Acta Anat., 85, 308-3i4.

Walls E. W., 1960. The microanatomy of muscle, in The structure and function of muscle, Academic Press, New York. 